\title{
Matrine pretreatment improves cardiac function in rats with diabetic cardiomyopathy via suppressing ROS/TLR-4 signaling pathway
}

\author{
Zhong-wei LIU ${ }^{1, *}$, Jun-kui WANG ${ }^{1}$, Chuan QIU², Gong-chang GUAN ${ }^{1}$, Xin-hong LIU $^{1}$, Shang-jian LI $^{1}$, Zheng-rong DENG ${ }^{1}$ \\ ${ }^{1}$ Department of Cardiology, Shaanxi Provincial People's Hospital, Xi'an 710000, China; ${ }^{2}$ Department of Biostatistics \& Bioinformatics, \\ School of Public Health \& Tropical Medicine, Tulane University, New Orleans, LA 70112, USA
}

\begin{abstract}
Aim: Matrine is an alkaloid from Sophora alopecuroides L, which has shown a variety of pharmacological activities and potential therapeutic value in cardiovascular diseases. In this study we examined the protective effects of matrine against diabetic cardiomyopathy (DCM) in rats.

Methods: Male SD rats were injected with streptozotocin (STZ) to induce DCM. One group of DCM rats was pretreated with matrine $\left(200 \mathrm{mg} \cdot \mathrm{kg}^{-1} \cdot \mathrm{d}^{-1}, \mathrm{po}\right)$ for 10 consecutive days before STZ injection. Left ventricular function was evaluated using invasive hemodynamic examination, and myocardiac apoptosis was assessed. Primary rat myocytes were used for in vitro experiments. Intracellular ROS generation, MDA content and GPx activity were determined. Real-time PCR and Western blotting were performed to detect the expression of relevant mRNAs and proteins.

Results: DCM rats exhibited abnormally elevated non-fasting blood glucose levels at 4 weeks after STZ injection, and LV function impairment at 16 weeks. The cardiac tissues of DCM rats showed markedly increased apoptosis, excessive ROS production, and activation of TLR-4/MyD-88/caspase-8/caspase-3 signaling. Pretreatment with matrine significantly decreased non-fasting blood glucose levels and improved LV function in DCM rats, which were associated with reducing apoptosis and ROS production, and suppressing TLR-4/MyD-88/caspase-8/caspase-3 signaling in cardiac tissues. Incubation in a high-glucose medium induced oxidative stress and activation of TLR-4/MyD-88 signaling in cultured myocytes in vitro, which were significantly attenuated by pretreatment with $\mathrm{N}$-acetylcysteine.

Conclusion: Excessive ROS production in DCM activates the TLR-4/MyD-88 signaling, resulting in cardiomyocyte apoptosis, whereas pretreatment with matrine improves cardiac function via suppressing ROS/TLR-4 signaling pathway.
\end{abstract}

Keywords: matrine; diabetes; heart; cardiomyocyte; cardiomyopathy; ROS; TLR-4; MyD-88; apoptosis; N-acetylcysteine

Acta Pharmacologica Sinica (2015) 36: 323-333; doi: 10.1038/aps.2014.127; published online 26 Jan 2015

\section{Introduction}

Hyperglycemia is one of the underlying metabolic abnormalities that characterizes diabetes mellitus ${ }^{[1]}$. The occurrence of both sustained hyperglycemia and various accompanying metabolic disorders may lead to the development of diabetic cardiomyopathy (DCM), one of the most common causes of disability and death among diabetic patients ${ }^{[2,3]}$. Hyperglycemia-induced oxidative stress plays a pivotal role in both the pathogenesis and the development of $\mathrm{DCM}^{[4-6]}$. Oxidative stress induced by the excess formation of reactive oxygen species (ROS) causes abnormal gene expression and abberant

\footnotetext{
* To whom correspondence should be addressed.

E-mail liuzhongweicn@gmail.com

Received 2014-05-23 Accepted 2014-10-14
}

signal transduction, and induces the activation of pathways that trigger cardiomyocyte apoptosis ${ }^{[5]}$. The resultant cardiac cell death induces the loss of cardiac contractile units and subsequently provokes cardiac remodeling, thereby playing an important role in the pathophysiological processes of $\mathrm{DCM}^{[2,7]}$.

The clinical application of the traditional Chinese herb, Sophora alopecuroides L, has a long history in China of effectively treating various diseases, including chronic liver disease, heart failure, and hypertension ${ }^{[8,9]}$. The herb's clinical effects were believed to be dependent on its active molecule, $\mathrm{C}_{15} \mathrm{H}_{24} \mathrm{~N}_{2} \mathrm{O}$, an alkaloid also known as matrine (Figure 1). Matrine has many biological activities and exerts many biological effects, such as anti-inflammation, anti-fibrosis, and immune regulation ${ }^{[10-12]}$. Although they are rarely mentioned, the anti-oxidant properties of matrine have been described in 


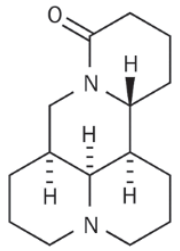

Figure 1. Chemical structure of matrine $\left(\mathrm{C}_{15} \mathrm{H}_{24} \mathrm{~N}_{2} \mathrm{O}\right)$.

previous studies $^{[13]}$. Matrine treatment exerted therapeutic benefits in cardiac injury and cardiac dysfunction ${ }^{[14]}$, and also induced cardiomyocyte apoptosis in hyperglycemia; although the underlying mechanisms of matrine's cytoprotective effects remain poorly understood.

Toll-like receptor-4 (TLR-4), a proximal signaling receptor responsible for triggering innate immune and inflammatory responses, is expressed in the heart; its activity correlates strongly with cardiac stress reactions ${ }^{[15-17]}$. Recent studies presented novel evidence that TLR-4 may contribute to myocardial apoptosis related to various cardiomyopathies in inflammation and oxidative stress, indicating that TLR-4 deficiency provides potent anti-apoptotic protection in myocytes ${ }^{[18-20]}$. Additionally, the excessive intracellular ROS generation induced by oxidative stress may activate the TLR- 4 signaling pathway $^{[21-23]}$.

Therefore, sustained hyperglycemia-induced myocyte apoptosis, a potent inducer of excessive ROS formation, is most likely related to the activation of the TLR-4 signaling pathway. More importantly, it is logical to speculate that matrine treatment alleviates cardiac cell apoptosis and cardiac dysfunction in rats with DCM, and that matrine's cytoprotective effect is mediated by suppressing the ROS/TLR-4 signaling pathway in vivo. In this study, we investigated the effects exerted by matrine on both myocyte apoptosis and cardiac dysfunction, as well as the underlying mechanisms involved in its cytoprotective effects, using an experimental DCM rat model. The results provide a new insight into potential cardioprotective therapies for DCM using matrine.

\section{Materials and methods}

\section{Animals, grouping and treatment}

Male Sprague-Dawley (SD) rats weighing 180-220 g were provided by the Experimental Animal Center of Xi'an Jiaotong University. All experimental procedures were carried out in accordance with the recommended guidelines for the care and use of laboratory animals issued by the Chinese Council on Animal Research. The protocol was approved by the ethics committee of Xi'an Jiaotong University.

Diabetes was induced in the male SD rats, using two consecutive intraperitoneal (ip) injections of streptozotocin (STZ, Sigma-Aldrich, St Louis, MO, USA, dissolved in $10 \mathrm{mmol} / \mathrm{L}$ sodium citrate buffer, $\mathrm{pH}=4.5$ ) at a dose of $60 \mathrm{mg} / \mathrm{kg}$ body weight ${ }^{[24]}$. For the present study, a diabetic model is defined as an experimental model characterized by a non-fasting blood glucose measurement of $16.7 \mathrm{mmol} / \mathrm{L}$ or higher, detected by two consecutive measurements, following the second STZ injection $^{[24,25]}$.

Thirty-two animals were divided equally and evenly into four groups: the control group (Ctrl), the matrine group (Mat), the DCM group (DCM) and the treatment group (DCM+Mat). In the Ctrl group, the rats received oral physiological saline (10 $\mathrm{mL} / \mathrm{kg}$ ) for 10 consecutive days; in the DCM group, the rats received oral physiological saline $(10 \mathrm{~mL} / \mathrm{kg})$ for 10 consecutive days before receiving STZ injections; in the Mat group, the rats received oral matrine (200 mg/ kg, Sigma-Aldrich) for 10 consecutive days ${ }^{[14]}$; in the DCM+Mat group, the rats received oral matrine $(200 \mathrm{mg} / \mathrm{kg})$ for 10 consecutive days before receiving STZ injections. Matrine was not sequentially administered before either STZ or the corresponding physiological saline injections in the Mat and the DCM+Mat groups. All rats had free access to water and food during the experimental protocol.

Eight weeks after STZ or corresponding physiological saline injections, hemodynamic parameters were measured and blood samples were collected. The hearts were removed, cleaned of fat, trimmed, and then immediately frozen in liquid nitrogen. All samples were kept at $-80^{\circ} \mathrm{C}$ until needed.

\section{Hemodynamic determination}

Rats were anesthetized via ip injections of chloral hydrate (10\%, $0.03 \mathrm{~mL} / \mathrm{kg}$ bodyweight). An invasive hemodynamic determination was performed using methods described in previous studies ${ }^{[26]}$. A Millar miniature catheter connected to a Medlab data acquisition system (Nanjing MedEase, Nanjing, China) was inserted into the left ventricle from the right carotid artery in order to record left ventricular systolic pressure (LVSP), left ventricular end-diastolic pressure (LVEDP), the maximal rate of increased pressure $\left(+\mathrm{LVd} P / \mathrm{d} t_{\text {max }}\right)$, and the maximal rate of decreased pressure $\left(-\mathrm{LVd} P / \mathrm{d} t_{\max }\right)$.

\section{Cell culture and treatment}

Primary myocytes were isolated from the hearts of 2-d-old neonatal SD rats provided by the Experimental Animal Center of Xi'an Jiaotong University. After the rats were sacrificed via cervical dislocation, their hearts were harvested and cleaned using cold physiological saline. The ventricular tissue was minced into small pieces, which were subsequently digested by Liberase $(4.5 \mathrm{mg} / \mathrm{mL}$, Roche, Basle, Switzerland). Following digestion, the cells were placed into a medium containing minimum essential medium (MEM, Gibco, New York, NY, USA) supplemented with $5 \%$ bovine calf serum (Gibco), 1.8 $\mathrm{mmol} / \mathrm{L} \mathrm{CaCl}_{2}, 2 \mathrm{mmol} / \mathrm{L}$ L-glutamine (Invitrogen, New York, $\mathrm{NY}$, USA), $10 \mathrm{mmol} / \mathrm{L}$ 2,3-butanedione monoxine (Invitrogen) and $100 \mathrm{U} / \mathrm{mL}$ penicillin-streptomycin mix (Invitrogen), on laminin-coated dishes, and incubated for $24 \mathrm{~h}$. The medium was replaced by fresh medium containing MEM, $0.1 \mathrm{mg} / \mathrm{mL}$ myocyte bovine serum albumin (Sigma-Aldrich), $2 \mathrm{mmol} / \mathrm{L}$ L-glutamine (Invitrogen) and $100 \mathrm{U} / \mathrm{mL}$ penicillin-streptomycin mix (Invitrogen). The cells were incubated at $37^{\circ} \mathrm{C}$ in a humidified incubator containing $5 \% \mathrm{CO}_{2}$.

When cell confluence exceeded $50 \%-60 \%$, the primary myo- 
cytes were incubated for $48 \mathrm{~h}$ with both a normal glucose (5.5 $\mathrm{mmol} / \mathrm{L})$ medium and a high-glucose $(33 \mathrm{mmol} / \mathrm{L})$ medium, which were designated as NG and HG, respectively. The high-glucose concentration was comparable to the serum glucose levels of diabetic rats. The cells incubated with highglucose medium for $48 \mathrm{~h}$ following $24 \mathrm{~h}$ of pretreatment with the ROS scavenger, $\mathrm{N}$-acetylcysteine (NAC, $1 \mathrm{mmol} / \mathrm{L}$, SigmaAldrich), were designated as HG+NAC.

\section{In situ apoptosis detection}

A terminal transferase UTP nick end labeling (TUNEL) assay was performed in order to detect the occurrence of apoptosis in cardiac tissue ${ }^{[27]}$. Briefly, paraffin-embedded heart tissue was sectioned at a thickness of $5 \mu \mathrm{m}$. The sections were subsequently deparaffinized and pre-treated with proteinase $\mathrm{K}$ $(20 \mu \mathrm{g} / \mathrm{mL}$, Sigma-Aldrich). After being soaked in phosphate buffer saline (PBS) for 15 minutes, the TUNEL assay was performed on each of the processed sections using a TUNEL assay kit (Roche), per the manufacturer's instructions. The samples were then observed under a microscope (BX51, Olympus, Tokyo, Japan).

\section{In situ ROS determination}

Dihydroethidium staining (DHE, Beyotime, Shanghai, China) was utilized in order to detect ROS generation in situ as previously described ${ }^{[28]}$. Fresh cardiac tissue was immersed in optimal cutting temperature (OCT) compound (Tissue-Tek, Torrance, CA, USA) and frozen on dry ice. Several 10- $\mu \mathrm{m}$ thick sections were obtained and placed on poly-lysine slides. The slides were then incubated with $10 \mu \mathrm{mol} / \mathrm{L} \mathrm{DHE}$ at $37^{\circ} \mathrm{C}$ for $45 \mathrm{~min}$ in a dark humidified chamber. For the in vitro evaluation, intracellular ROS generation among the cultured myocytes was determined via 2,7-dichlorofluorescein (DCFH-DA, Molecular Probes, New York, NY, USA) staining. After being washed by PBS, the myocytes were incubated with DCFH-DA at $37^{\circ} \mathrm{C}$ for $30 \mathrm{~min}$ in a dark humidified chamber. Fluorescent images were obtained using an inverted fluorescence microscope (TE2000U, Nikon, Tokyo, Japan) and analyzed using Image-Pro Plus 5.0 software.

\section{Oxidative and anti-oxidative status evaluation}

Heart homogenate $(10 \%, w / v)$ was prepared with PBS $(\mathrm{pH}$ 7.4 ) and centrifuged at $12000 \times g$ for $15 \mathrm{~min}$ at $4^{\circ} \mathrm{C}$. The supernatant was collected and frozen at $-80^{\circ} \mathrm{C}$ in aliquots until needed for biochemical assays. Both the malondialdehyde (MDA) level and glutathione peroxidase (GPx) activity were measured according to instructions for their respective commercial assay kits (Nanjing Jiancheng Bioengineering Institute, Nanjing, China), using spectrophotometry ${ }^{[29]}$.

\section{Real-time polymerase chain reaction (PCR)}

Real-time PCR was utilized in order to examine the expression of TLR-4 and MyD-88 at the transcriptional level. According to the manufacturer's instructions, total RNA was extracted from either cardiac tissue samples or cultured myocytes using either an RNAfast 200 Kit (Fastagen, Shanghai, China) or
TRI Reagent RNA Isolation Reagent (Sigma-Aldrich), before being reversely transcribed into cDNA using PrimeScript RT Master Mix (TaKaRa, Otsu, Japan). SYBR Premix Ex Taq ${ }^{\mathrm{TM}}$ II (TaKaRa) was then used to perform real-time PCR. Primers for TLR-4 and MyD-8 ${ }^{[30]}$ were designed and synthesized by TaKaRa as shown in Table 1. The relative levels of mRNA were normalized to GADPH and calculated via the $\triangle$ cycle threshold equation $\left(\Delta \mathrm{C} t=\mathrm{C} t_{\text {Target }}-\mathrm{C} t_{\mathrm{GAPDH}}\right)$, using Bio-Rad IQ5 software (Version 1.0, Bio-Rad, hercules, CA, USA).

Table 1. Sequences of primers for real-time PCR.

\begin{tabular}{llc}
\hline Gene & \multicolumn{1}{c}{ Sequence } & Size (bp) \\
\hline TLR-4 & $\begin{array}{l}\text { Forward 5'-AGACATCCAAAGGAATACTGCAA-3' } \\
\text { Myd-88 }\end{array}$ & 180 \\
& $\begin{array}{l}\text { Reverse 5'-GCCTTCATGTCTATAGGTGATGC-3' } \\
\text { Forward 5'-CTCCATTCCTCCTCCAGACACT-3' }\end{array}$ & 196 \\
GAPDH & $\begin{array}{l}\text { Reverse 5'-AAGGAGAGGCAGTTTGGCTTC-3' } \\
\text { Forward 5'-CATCACTGCCACTCAGAAGA-3' }\end{array}$ & 330 \\
& Reverse 5'-TGAAGTCACAGGAGACAACC-3' & \\
\hline
\end{tabular}

\section{Western blotting assay}

According to the manufacturer's instructions, heart samples and cultured myocytes were lysed and homogenized in a RIPA lysis buffer system (Santa Cruz, Dallas, TX, USA) with PMSF (Santa Cruz) for $30 \mathrm{~min}$, on ice. Supernatants were collected via centrifugation at $12500 \times \mathrm{g}$ for $10 \mathrm{~min}$ at $4^{\circ} \mathrm{C}$. The total protein concentration in the supernatants was determined using a BCA protein assay kit (Santa Cruz). The sample protein was treated with $1 \times$ SDS-PAGE loading buffer before being separated by electrophoresis in 10\% SDS-polyacrylamide gel and transferred onto a polyvinylidene fluoride (PVDF) membrane. Antibodies to TLR-4 (CST, Beverly, MA, USA), MyD-88 (CST), pro-caspase-8 (Abcam, Cambridge, MA, USA), caspase-8 P18 (Santa Cruz), pro-caspase-3 (Abcam), caspase-3 P17 (Santa Cruz), and GAPDH (Santa Cruz) were used to detect the immunoblots at $4{ }^{\circ} \mathrm{C}$ overnight. After being washed, the membranes were incubated with horseradish peroxidase conjugated secondary antibodies (Santa Cruz). Membranes were subsequently developed using Western Blotting Luminal Reagent (Santa Cruz) for detection.

\section{Statistical analysis}

Data in this study were expressed as the mean \pm SD and analyzed by SPSS (Version 17.0) software. One-way ANOVA was utilized to analyze the differences between means. A $P$-value $<0.05$ was statistically significant.

\section{Results}

Diabetic and DCM animal model establishment

As shown in Figure 2B, all rats in the DCM and DCM+Mat groups exhibited abnormally elevated non-fasting blood glucose levels; non-fasting blood glucose concentrations increased significantly in both the DCM and DCM+Mat groups, whereas 
A
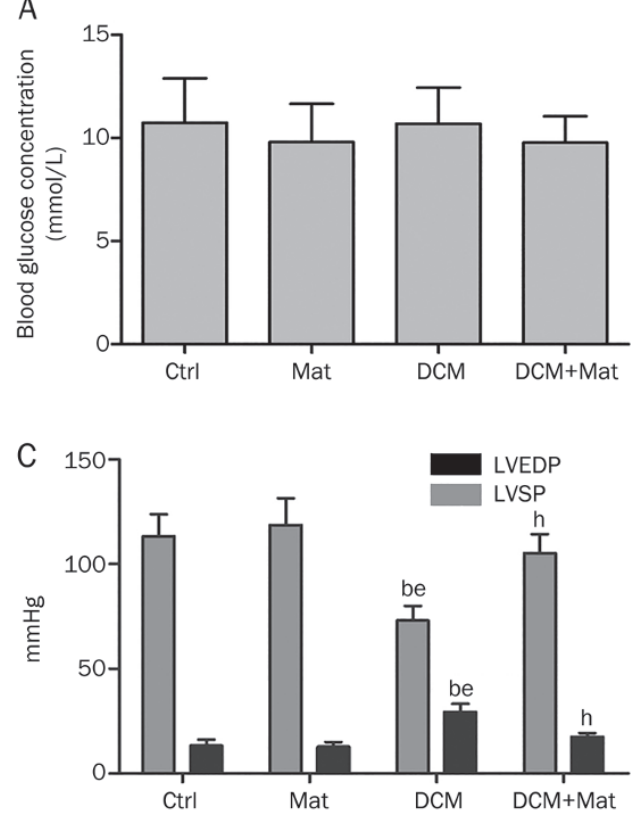

B
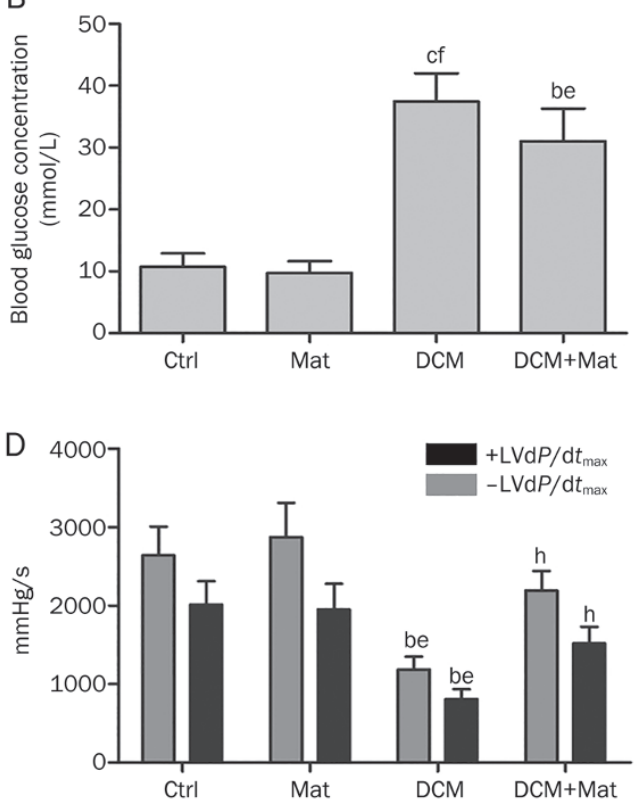

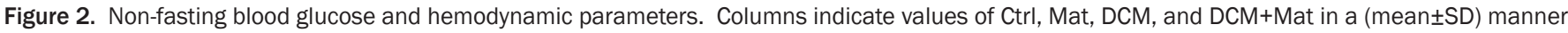

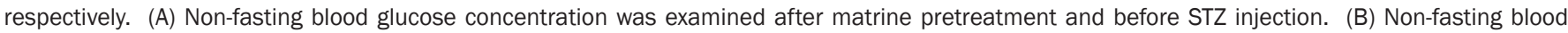

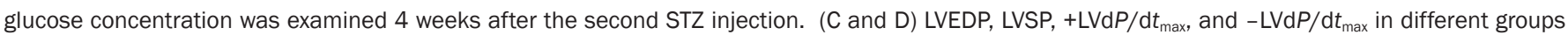

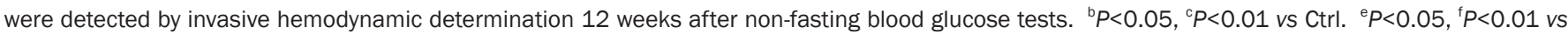
Mat. ${ }^{\mathrm{h}} \mathrm{P}<0.05$ vs DCM.

no significant changes were noted in the non-fasting blood glucose concentrations of the Ctrl and Mat groups. The results of the evaluation of cardiac function based on hemodynamic parameters are included in Figures 2C and 2D. A significantly increased LVEDP was observed; however, decreases in LVSP, $+\mathrm{LVd} P / \mathrm{d} t_{\max }$, and $-\mathrm{LVd} P / \mathrm{d} t_{\max }$ were observed in the DCM group compared with the Ctrl group and the Mat group. However, in the DCM+Mat group, matrine administration significantly decreased LVEDP and improved LVSP, +LVdP/ $\mathrm{d} t_{\max }$, and $-\mathrm{LVd} P / \mathrm{d} t_{\max }$ compared with the DCM group.

\section{Effects of matrine administration on myocyte apoptosis in the setting of DCM}

Myocyte apoptotic death was measured via TUNEL staining of formalin-fixed cardiac tissues. As demonstrated in Figure 3, the DCM group displayed more TUNEL-positive cells compared with the Ctrl group and the Ctrl+Mat group. By contrast, the administration of matrine attenuated myocyte apoptosis significantly in the DCM+Mat group.

Effects of matrine administration on cardiac ROS generation, MDA levels and GPx activity

As shown in Figure 4A, the intracellular ROS content of frozen cardiac tissue sections from each group was examined using an inverted fluorescence microscope. DHE fluorescence intensity, a relatively specific indicator of ROS, was significantly stronger in the DCM group than in both the Ctrl group and the Mat group. However, DHE intensity was decreased in the DCM+Mat group as a result of matrine treatment. Figure
4B depicts the detected cardiac MDA levels of each group, as MDA levels are considered direct markers of oxidative stress. A significantly increased MDA level was noted in the DCM group, a level that was substantially reduced by matrine administration. Cardiac GPx activity is shown in Figure 4C. Compared with the Ctrl and the Mat groups, cardiac GPx activity was significantly decreased in the DCM group but was increased by matrine treatment in the DCM+Mat group.

ROS/TLR-4/MyD-88 signaling activation in high-glucose incubated myocytes and the effects of matrine administration on the TLR-4/MyD-88 signaling pathway

As demonstrated in Figure 5, compared with the NG group, the intracellular ROS levels and the mRNA and protein expression levels of both TLR-4 and MyD-88 increased significantly in the HG group. Correspondingly, the expression levels of cleaved caspase- 8 and cleaved caspase- 3 were elevated in the HG group compared with the NG group. However, in the HG+NAC group, NAC pretreatment significantly reduced intracellular ROS levels, as well as the expression levels of TLR-4, MyD-88, cleaved caspase-8, and cleaved caspase-3 in high-glucose incubated primary myocytes. As shown in Figure 6, compared with the Ctrl and Mat groups, dramatic increases in the levels of TLR-4 and MyD-88 were noted at both the transcriptional and translational levels in the setting of DCM. Additionally, the expression levels of both downstream cleaved caspase- 8 and cleaved caspase- 3 were also elevated in the setting of DCM. Also demonstrated in Figure 6, matrine treatment significantly reduced the expression levels 

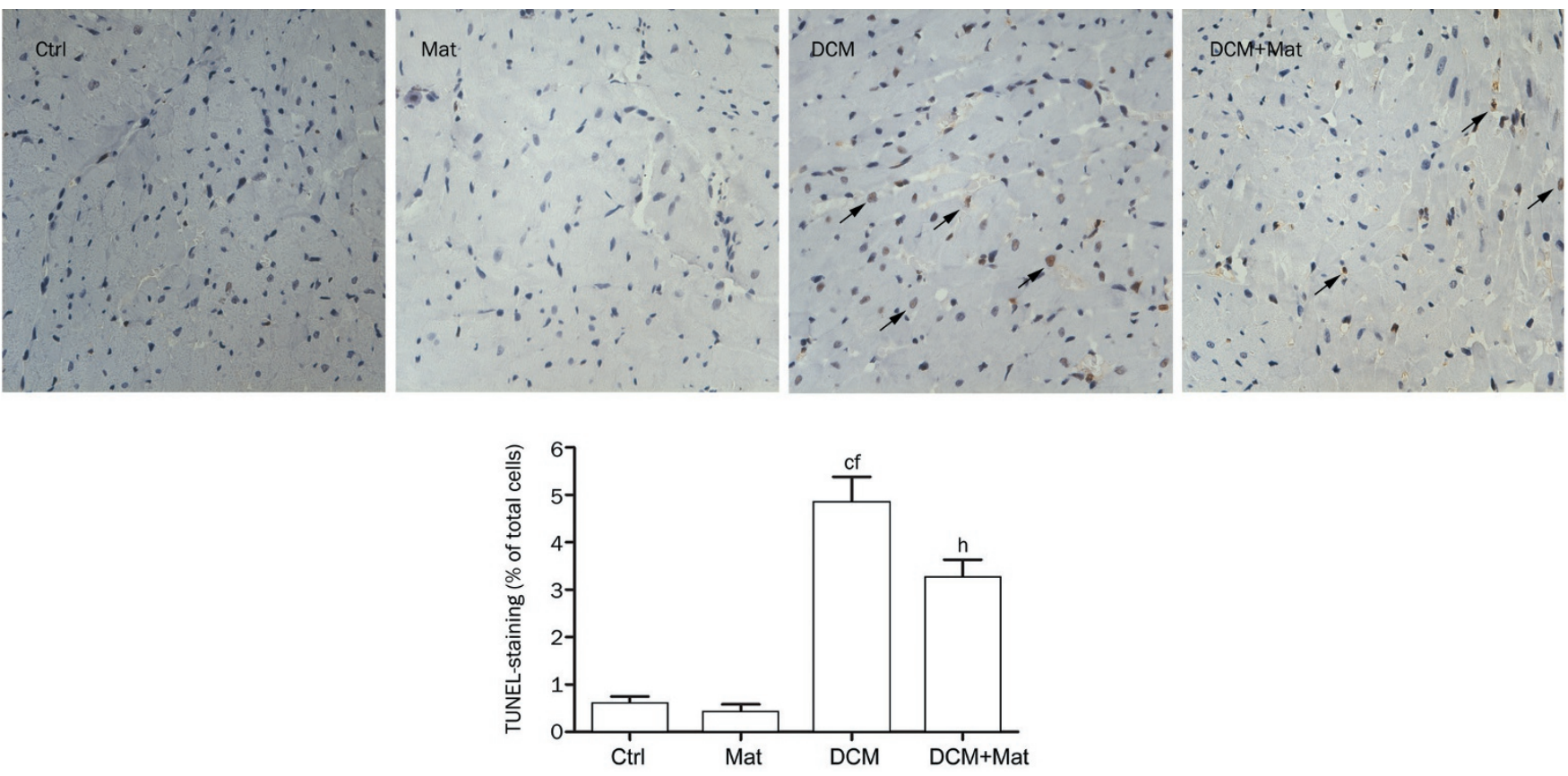

Figure 3. Matrine administration attenuated myocytes apoptosis in cardiac tissue from rats with DCM. The upper panel represents TUNEL assay of paraffin-embedded myocardial tissue slides in Ctrl, Mat, DCM, and DCM+Mat. Representative photomicrographs of TUNEL positive staining in myocytes are indicated by black arrows. The lower panel shows the quantification of TUNEL-positive (yellow-brown stained) cardiomyocytes. Columns indicate detected values of Ctrl, Mat, DCM, and DCM+Mat respectively in (mean \pm SD) manner. ${ }^{\mathrm{c}} P<0.01$ vs Ctrl. ${ }^{\mathrm{f}} P<0.01$ vs Mat. ${ }^{\mathrm{h}} P<0.05$ vs DCM.

of TLR-4, MyD-88, cleaved caspase-8, and cleaved caspase-3 in the DCM+Mat group compared with the DCM group.

\section{Discussion}

DCM, which is accompanied by disordered glucose metabolism, is defined as ventricular dysfunction and structural abnormalities independent of hypertensive heart disease, valvular heart disease, coronary artery disease and other cardiovascular diseases in diabetic individuals ${ }^{[31]}$. DCM is usually characterized primarily by the presence of diastolic dysfunction, which may precede the development of systolic dysfunction ${ }^{[31,32]}$. In our study, diabetes was induced in rats via the ip injection of STZ, which exerts preferential toxicity on the insulin-producing beta cells of the pancreas. DCM was confirmed in diabetic rats based on evidence of decreased LV systolic and diastolic myocardial performance, changes that are associated with substantial TUNEL-positive staining myocytes in cardiac tissue and are consistent with the results of previous studies ${ }^{[33,34]}$. Early responses of the heart to sustained hyperglycemia include metabolic disorders, subcellular defects, aberrant gene expression, and cell apoptosis ${ }^{[2]}$. A previous study also suggested that cardiac myocytes were more susceptible to diabetes-induced cell apoptosis or death ${ }^{[35]}$. Cardiomyocyte apoptosis is one of hallmarks of DCM, in both the pathogenesis and the development of impaired cardiac function ${ }^{[2,7,36]}$. The loss of contractile units reduces cardiac function and triggers myocyte hypertrophy, which contributes to both the development and subsequent progression of DCM.

Oxidative stress has been linked to both the onset of dia- betes and to its complications ${ }^{[5]}$. Regarding the destructive aspects of oxidative stress, excess ROS formation has been linked to the activation of apoptotic cascades in different cell types ${ }^{[37,38]}$. Moreover, accumulating evidence suggests that hyperglycemia causes apoptosis in cardiomyocytes by stimulating ROS production ${ }^{[39-41]}$. Therefore, excessive ROS represent critical and central mediators of the complicated cascades associated with myocardial cell apoptosis in the setting of DCM. It is believed that hyperglycemia either directly or indirectly participates in ROS production via glucose autoxidation, alterations in the sorbitol (polyol) pathway, the formation of advanced glycation end products (AGEs), and the activation of both protein kinase $\mathrm{C}$ (PKC) and nicotinamide adenine dinucleotide phosphate (NADPH)-oxidase ${ }^{[42,43]}$. More importantly, a previous in vitro study demonstrated that high glucose concentrations induce TLR-4 expression via PKC by stimulating NADPH oxidase ${ }^{[44]}$. Unregulated TLR-4 activation was also observed in the monocytes, the endothelial cells, and the islets of both diabetic patients and mice ${ }^{[45-47]}$. Indeed, the results of the in vitro experiments performed in this work were consistent with this hypothesis. Oxidative stress was stimulated by high-glucose incubation, as demonstrated by the elevated intracellular ROS levels noted during the study. Meanwhile, the activation of TLR-4/MyD-88/caspase-8/ caspase-3 signaling was also found in high-glucose incubated myocytes. NAC exhibited its anti-oxidant effect by eliminating intracellular ROS in high-glucose incubated myocytes. As a result, the TLR-4/MyD-88/caspase-8/caspase-3 signaling pathway was also inhibited, indicating that TLR-4/MyD-88/ 
A

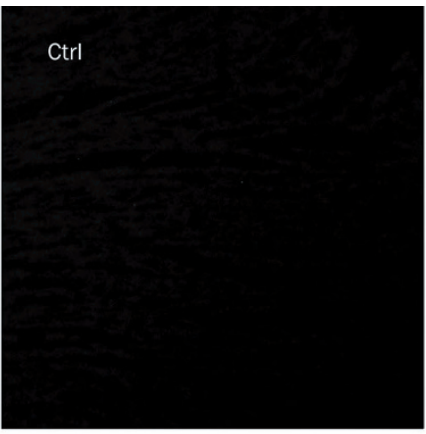

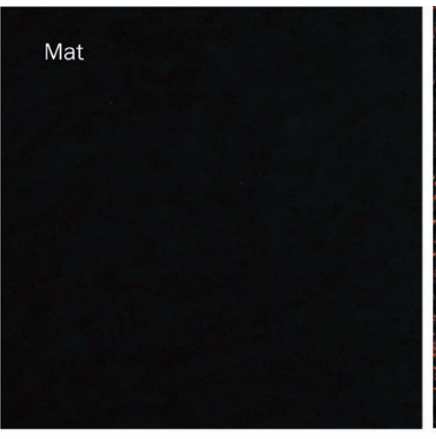
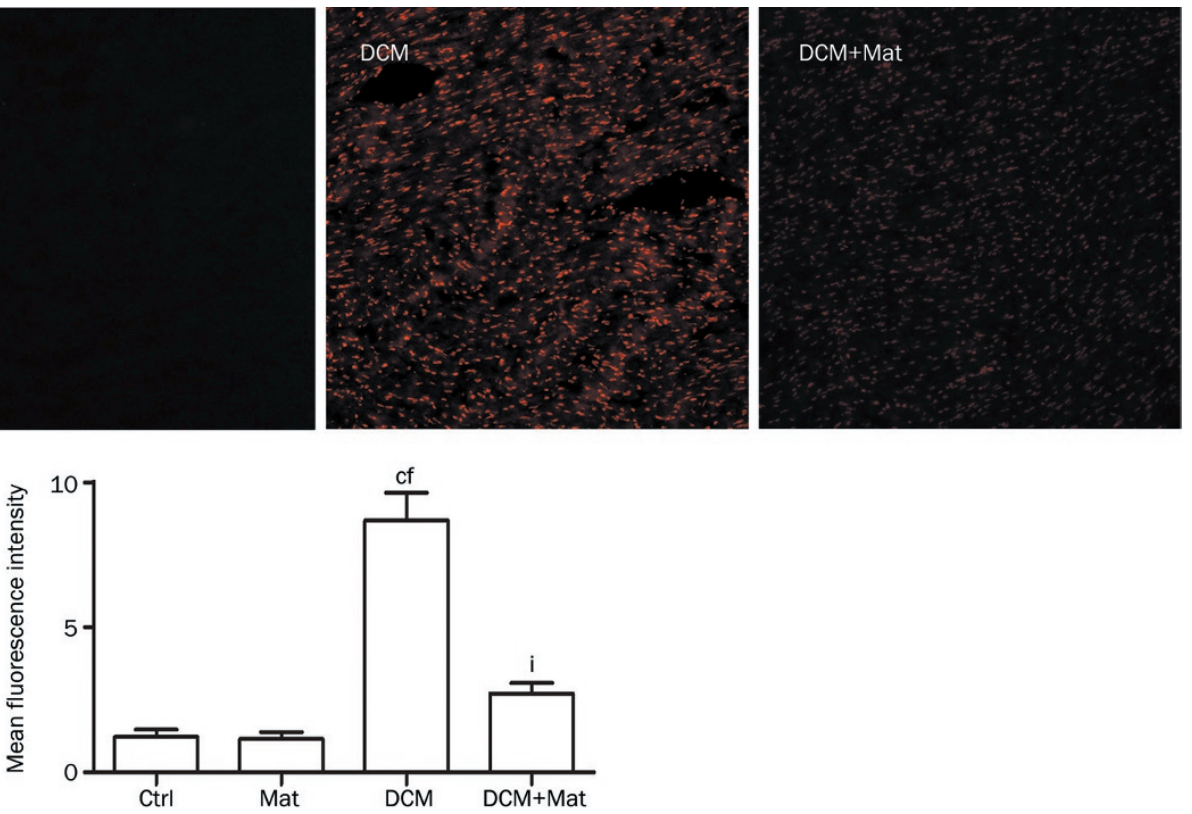

B

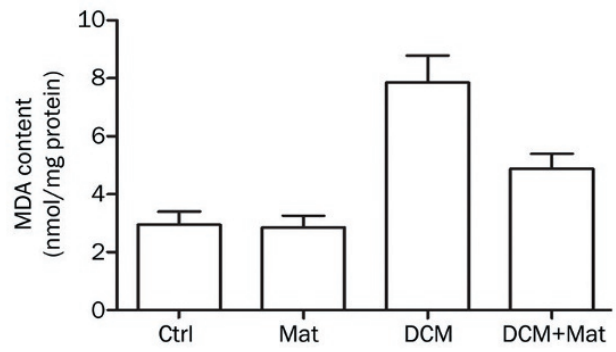

C

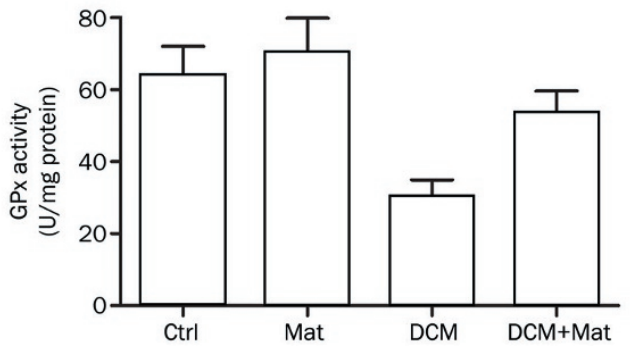

Figure 4. Matrine administration improved redox balance in cardiac tissue from DCM rats. (A) Intracellular ROS detection by DHE staining. The upper part shows fluorescence microscopic images of cardiac frozen sections in Ctrl, Mat, DCM, and DCM+Mat. The lower part demonstrates the values of mean fluorescence intensity which stands for the level of intracellular ROS. (B) Determination of MDA content in cardiac tissue by spectrophotometric method in each group. (C) Determination of GPx activity in cardiac tissue by spectrophotometric method in each group. Values indicated by columns in graph are presented as the mean \pm SD. ${ }^{c} P<0.01$ vs Ctrl. ${ }^{f} P<0.01$ vs Mat. ${ }^{i} P<0.01$ vs DCM.

caspase-8/caspase-3 signaling was activated in the setting of intensified oxidative stress. During the in vivo study, following STZ treatment, the serum MDA level, which is a direct marker of oxidative stress, was significantly increased in the cardiac tissues of the rats with DCM. As shown using DHE staining, higher intracellular ROS production in cardiac tissue, as well as a higher TLR-4/MyD-88 expression level and a larger amount of TUNEL-positive myocytes, was observed following exposure to sustained hyperglycemia, a finding that was not noted among the non-diabetic hearts.

TLR-4 is a key member of TLR family, which binds to components of pathogens and participates in innate immune responses in mammals. TLR- 4 is expressed in multiple types of cardiac cells, including endothelial cells, smooth muscle cells and cardiomyocytes. Emerging evidence indicates that TLR-4 is not only an important part of the innate immune system but is also involved in cardiac dysfunction and damage in the setting of inflammation and oxidative stress ${ }^{[48]}$. Increased TLR-4 expression was noted in myocytes isolated from both humans and experimental animals with various types of cardiomyopathies $^{[49]}$. In a mouse model of myocardial infarction (MI), TLR-4 controlled signaling pathways were activated as a result of the cardiac stress caused by ischemia. Additionally, TLR-4 deficiency led to both increased cell survival and to improved LV function, changes accompanied by the alleviation of apoptosis in the heart ${ }^{[48]}$. In a doxorubicin-induced cardiomyopathy mouse model, TLR4-deficiency improved left ventricular function due to the suppression of oxidative and inflammatory stress responses and to reduced myocyte apoptosis ${ }^{[18]}$. Similar findings were observed in a trastuzumabinduced cardiomyopathy model in mice ${ }^{[16]}$. MyD-88 is a key adaptor protein involved in TLR-4 signal transduction and triggers both the nuclear translocation and the activation of nuclear factor kappa B (NF-kB), as well as the transcription of inflammatory cytokine genes and the activation of mitogenactivated protein kinases (MAPKs), leading to cell apoptosis $^{[50]}$. The blockade of MyD-88, an important component of the TLR-4 signaling pathway, significantly improved cardiac 
A
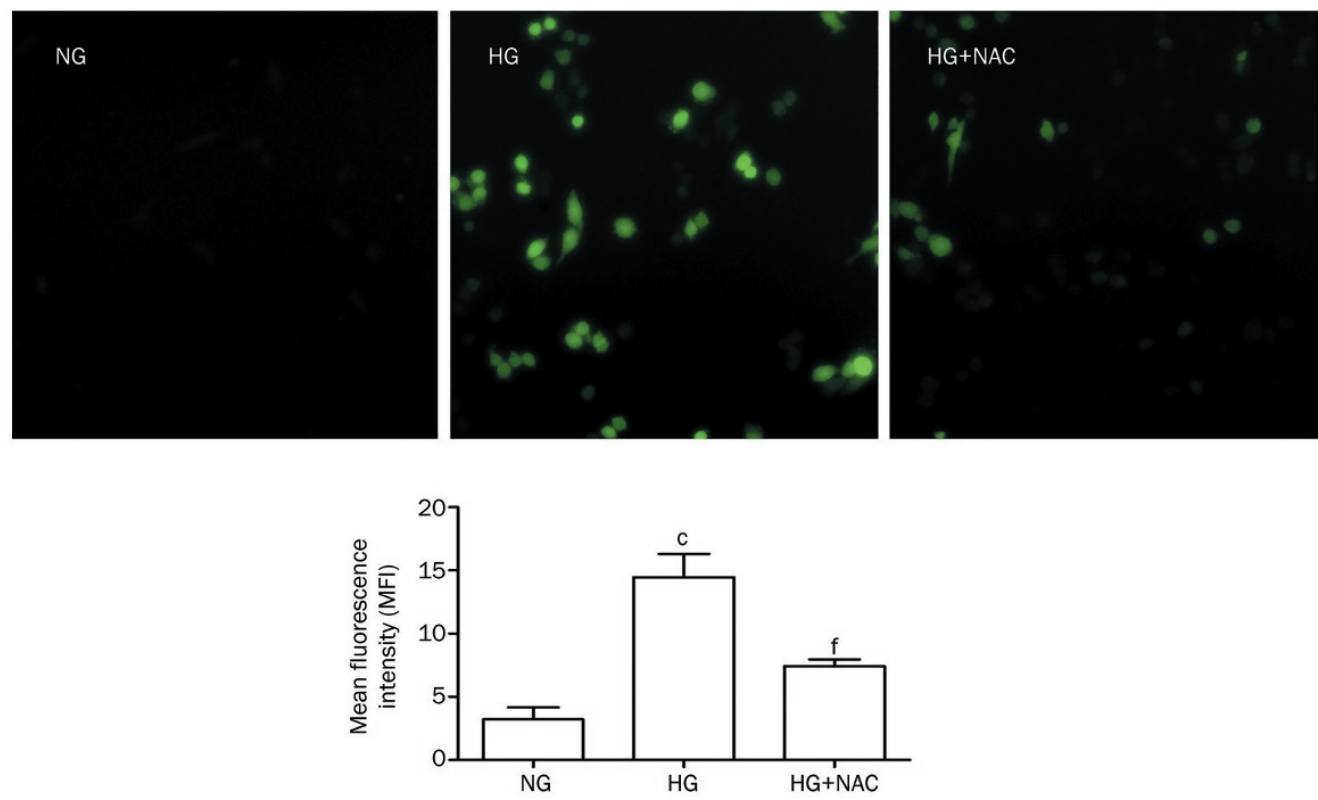

Figure 5A. Oxidative stress-induced TLR-4/MyD-88 signaling activation in high-glucose incubated primary myocytes. (A) The upper part shows the fluorescence microscopic images of ROS detection by DCFH-DA staining in NG, HG, and HG+NAC respectively. The lower part demonstrated the quantification of intracellular ROS by analyzing mean fluorescent intensity.

function, reduced cardiac myocyte apoptosis and ultimately attenuated cardiac hypertrophy in vivo ${ }^{[51]}$. In the current study, we found that the TLR-4/MyD-88 pathway was significantly up-regulated in cardiac tissue exposed to sustained hyperglycemia in rats with DCM. Meanwhile, molecules such as caspase- 3 and caspase- 8 were also activated as part of the caspase cascade. Although the specific mechanism underlying how TLR-4 signaling triggers apoptosis is relatively complex, it is believed to be the result of an interaction with the Fas-associated death domain protein (FADD) through MyD- $88^{[52,53]}$; TLR-4 recruits and activates caspase-8, the initiator of the apoptotic cascade ${ }^{[54]}$. The activated caspase- 8 both cleaves and directly activates pro-caspase- 3 , which ultimately leads to cell death ${ }^{[55]}$.

ROS generation plays a critical role in the upregulation of the TLR-4 signaling pathway; intracellular ROS production up-regulates TLR-2/4 expression, leading to the release of pro-inflammatory cytokines ${ }^{[56]}$; the inhibition of oxidative stress-induced ROS production significantly suppresses the translocation of TLR-4 into lipid rafts, as well as the upregulation of TLR-4 expression and TLR-4 mediated cytokine production $^{[22,23]}$, findings that indicate that ROS production may play a vital role in modulating the TLR-4 signaling pathway. Furthermore, it is likely that excessive ROS formation and TLR-4/MyD-88 pathway activation cooperatively induce apoptotic events in cardiomyocytes under both hyperglycemic conditions and following high-glucose incubation ${ }^{[19,57]}$. In the present study, we found that the increased levels of TLR-4 induced myocyte apoptosis were associated with the accumulation of intracellular ROS in the cardiac tissue of rats with DCM. Therefore, it may be that sustained hyperglycemia induced ROS production was initially recognized as a harmful signal by TLR-4. The activation of TLR-4 and its down-stream mediators triggered a subsequent caspase cascade that eventually led to cardiomyocyte apoptosis in diabetic rats.

The results of this study also indicate matrine's protective effect against myocyte apoptosis, which was responsible for the impaired cardiac function noted in the setting of DCM. The administration of matrine exerted distinct positive effects on LV function, as evidenced by an overall increase in LVSP, $+\mathrm{LVd} P / \mathrm{d} t_{\max }$ and $-\mathrm{LVd} P / \mathrm{d} t_{\max }$, changes accompanied by an overall decrease in LVEDP; mechanically, the activity of the TLR-4 pathway and the number of TUNEL positive myocytes decreased dramatically. ROS are important contributors to the pathogenesis and the development of DCM; ROS scavenging by antioxidants prevented hyperglycemia-induced cardiac cell death and the subsequent development of compensatory hypertrophy ${ }^{[39]}$. In this study, we observed that matrine administration resulted in an apparent increase in GPx activity and a decrease in ROS formation in diabetic hearts. More importantly, matrine treatment also decreased the expression of both TLR-4 and MyD-88, and down-regulated the activity levels of both caspase- 8 and caspase-3, as well. These findings are illustrative of matrine's cardioprotective effect, which it exerts by reducing myocyte apoptosis in the setting of DCM. This anti-apoptotic effect correlated strongly with the deactivation of the TLR-4/MyD-88/caspase signaling pathway. These changes contributed to the cytoprotective effects of matrine against both the development and the progression of DCM; however, more specific information regarding the mechanisms underlying these effects still needs to be uncovered.

\section{Conclusion}

Excessive ROS production induces the activation of the 

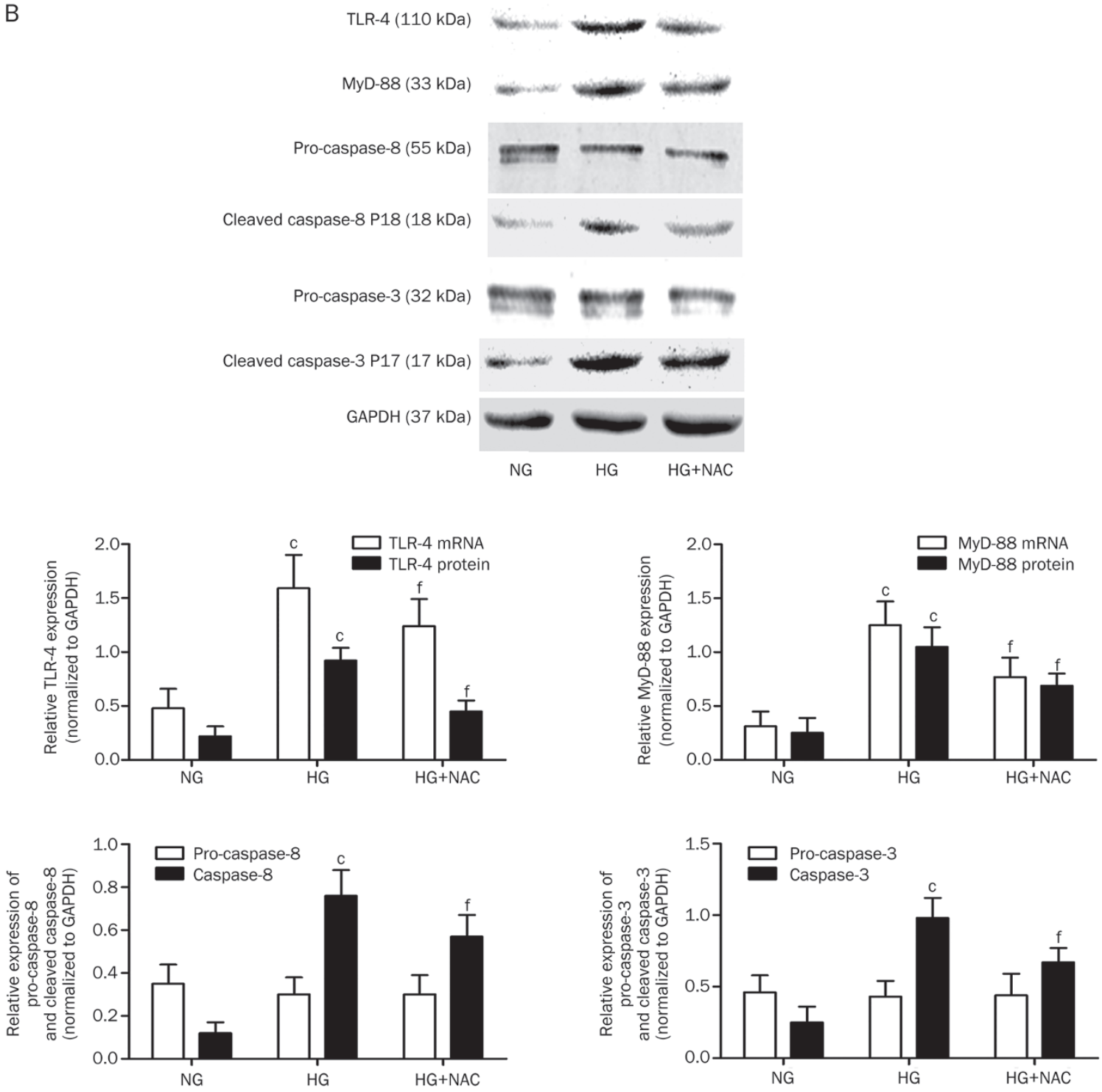

Figure 5B. (B) The upper part demonstrated the results of Western blotting assay in primary myocytes. Immunoblots of TLR-4, MyD-88, pro-caspase-3, cleaved caspase-3, pro-caspase-8, cleaved caspase-8, and GAPDH in NG, HG, and HG+NAC are shown respectively. Columns at the lower part of (B) represents the quantification of relative expression levels of TLR-4, MyD-88, pro-caspase-8, cleaved caspase-8, pro-caspase-3, and cleaved caspase-3 in $\mathrm{NG}, \mathrm{HG}$, and $\mathrm{HG}+\mathrm{NAC}$ respectively. Values are presented as the mean $\pm \mathrm{SD}$. ${ }^{\mathrm{c}} P<0.01$ vs $\mathrm{NG}$. ${ }^{\mathrm{f}} P<0.01$ vs $\mathrm{HG}$.

TLR-4/MyD-88 signaling pathway, leading to cardiomyocyte apoptosis in the setting of DCM.

Matrine exerts its anti-apoptotic effects by modulating ROSinduced TLR-4/MyD-88 signaling activation and may represent a promising cardioprotective therapy for DCM.

\section{Acknowledgements}

We thank Ms Ning JING for her expert technical assistance.

\section{Author contribution}

Zhong-wei LIU and Chuan QIU carried out the study's primary experiments and contributed to the writing of the manuscript; Jun-kui WANG participated in the writing of the manuscript; Zheng-rong DENG and Shang-jian LI designed the artwork for the manuscript's figures; Gong-chang GUAN and Xin-hong LIU performed the statistical analysis.

\section{References}

1 American Diabetes Association Editors. Diagnosis and classification of diabetes mellitus. Diabetes Care 2009; 32 Suppl 1: S62-7.

2 Cai L, Kang YJ. Cell death and diabetic cardiomyopathy. Cardiovasc Toxicol 2003; 3: 219-28.

3 Fang ZY, Prins JB, Marwick TH. Diabetic cardiomyopathy: evidence, mechanisms, and therapeutic implications. Endocr Rev 2004; 25: 543-67.

4 Giacco F, Brownlee M. Oxidative stress and diabetic complications. Circ Res 2010; 107: 1058-70.

5 Cai L, Kang YJ. Oxidative stress and diabetic cardiomyopathy: a brief review. Cardiovasc Toxicol 2001; 1: 181-93. 

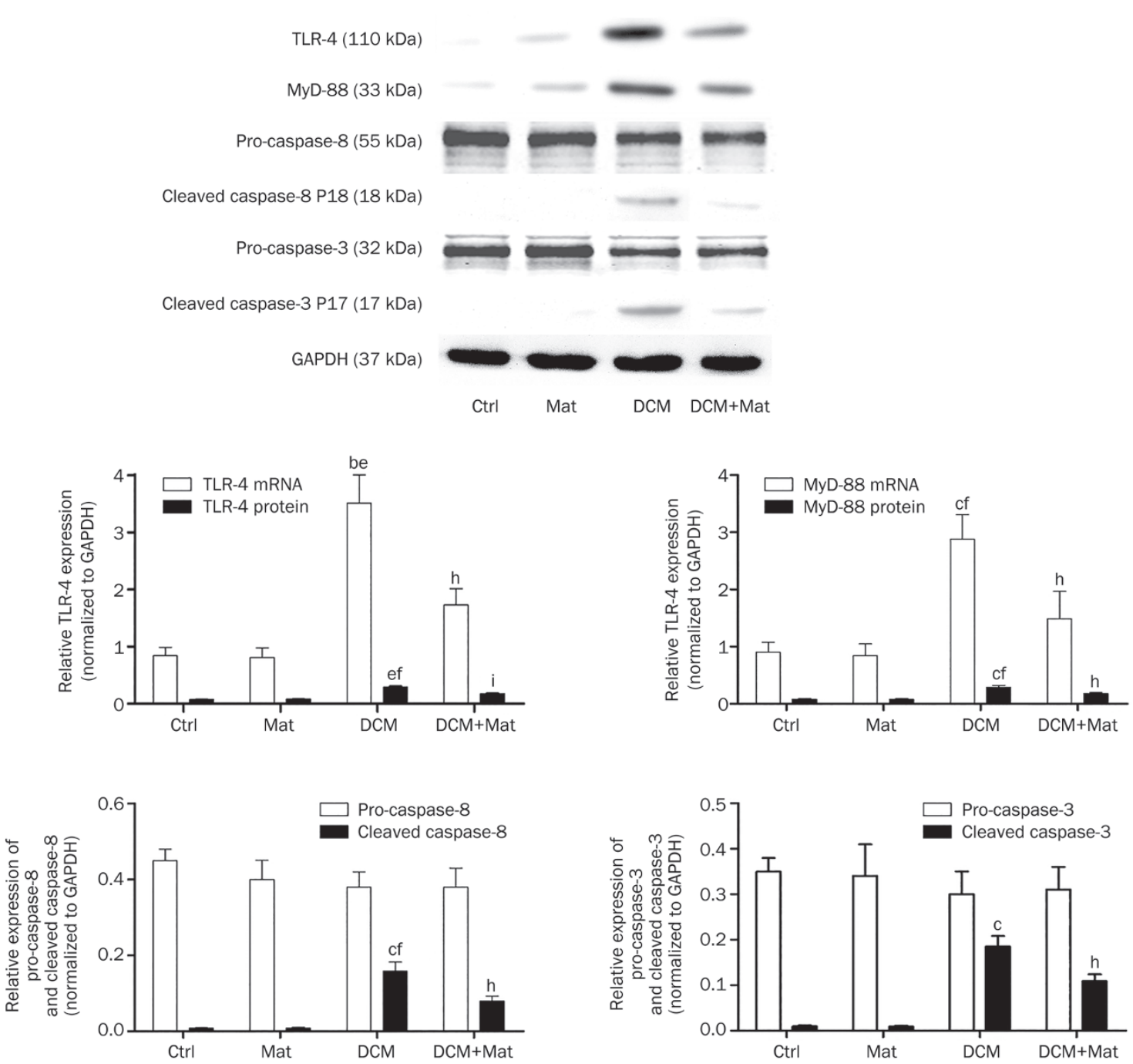

Figure 6. Effects of matrine administration on TLR-4/MyD-88 signaling in cardiac tissue from DCM rats. The upperpart shows the measurement of TLR-4, MyD-88, pro-caspase-8, cleaved caspase-8, pro-caspase-3, and cleaved caspase-3 expression by Western blotting. The immunoblots of TLR-4, MyD-88, Caspase-8 P18, Caspase-3 P17, and GAPDH in cardiac tissue from Ctrl, Mat, DCM, and DCM+Mat groups are shown respectively. Columns at the lower part of this figure stand for the relative expression levels of TLR-4, MyD-88, Caspase-8 P18, and Caspase-3 P17 (normalized to GAPDH) in described groups. Values are presented as the mean \pm SD. ${ }^{b} P<0.05,{ }^{c} P<0.01$ vs $C$ trl. ${ }^{e} P<0.05,{ }^{f} P<0.01$ vs Mat. ${ }^{\mathrm{h}} P<0.05,{ }^{\mathrm{i}} P<0.01$ vs $D C M$.

6 Khullar M, Al-Shudiefat AA, Ludke A, Binepal G, Singal PK. Oxidative stress: a key contributor to diabetic cardiomyopathy. Can J Physiol Pharmacol 2010; 88: 233-40.

7 Cai L, Wang Y, Zhou G, Chen T, Song Y, Li X, et al. Attenuation by metallothionein of early cardiac cell death via suppression of mitochondrial oxidative stress results in a prevention of diabetic cardiomyopathy. J Am Coll Cardiol 2006; 48: 1688-97.

8 Zhang F, Wang X, Tong L, Qiao H, Li X, You L, et al. Matrine attenuates endotoxin-induced acute liver injury after hepatic ischemia/reperfusion in rats. Surg Today 2011; 41: 1075-84.

9 Lao Y. Clinical study on effect of matrine injection to protect the liver function for patients with primary hepatic carcinoma after trans-artery chemo-embolization (TAE). Zhong Yao Cai 2005; 28: 637-8.

10 Lei ZL, Liu XJ, Ma JX, Zhu J. Effects of matrine on airway inflammation and early airway remodeling in asthmatic mice. Zhonghua Jie $\mathrm{He} \mathrm{He}$ Hu Xi Za Zhi 2009; 32: 165-70.
11 Gao HY, Li GY, Lou MM, Li XY, Wei XY, Wang JH. Hepatoprotective effect of matrine salvianolic acid $B$ salt on carbon tetrachlorideinduced hepatic fibrosis. J Inflamm (Lond) 2012; 9: 16.

12 Li T, Wong VK, Yi XQ, Wong YF, Zhou H, Liu L. Matrine induces cell anergy in human Jurkat $T$ cells through modulation of mitogenactivated protein kinases and nuclear factor of activated T-cells signaling with concomitant up-regulation of anergy-associated genes expression. Biol Pharm Bull 2010; 33: 40-6.

13 Zhang B, Liu ZY, Li YY, Luo Y, Liu ML, Dong HY, et al. Antiinflammatory effects of matrine in LPS-induced acute lung injury in mice. Eur J Pharm Sci 2011; 44: 573-9.

14 Li X, Zhou R, Zheng P, Yan L, Wu Y, Xiao X, et al. Cardioprotective effect of matrine on isoproterenol-induced cardiotoxicity in rats. J Pharm Pharmacol 2010; 62: 514-20.

15 Oyama J, Blais C, Jr., Liu X, Pu M, Kobzik L, Kelly RA, et al. Reduced myocardial ischemia-reperfusion injury in toll-like receptor 4-deficient 
mice. Circulation 2004; 109: 784-9.

16 Yousif NG, Al-Amran FG. Novel Toll-like receptor-4 deficiency attenuates trastuzumab (herceptin) induced cardiac injury in mice. BMC Cardiovasc Disord 2011; 11: 62.

17 Chao W. Toll-like receptor signaling: a critical modulator of cell survival and ischemic injury in the heart. Am J Physiol Heart Circ Physiol 2009; 296: H1-12.

18 Riad A, Bien S, Gratz M, Escher F, Westermann D, Heimesaat MM, et al. Toll-like receptor-4 deficiency attenuates doxorubicin-induced cardiomyopathy in mice. Eur J Heart Fail 2008; 10: 233-43.

19 Zhang $\mathrm{Y}$, Peng T, Zhu H, Zheng X, Zhang X, Jiang N, et al. Prevention of hyperglycemia-induced myocardial apoptosis by gene silencing of Toll-like receptor-4. J TransI Med 2010; 8: 133.

20 Zhao P, Wang J, He L, Ma H, Zhang X, Zhu X, et al. Deficiency in TLR4 signal transduction ameliorates cardiac injury and cardiomyocyte contractile dysfunction during ischemia. J Cell Mol Med 2009; 13: 1513-25.

21 Sarir H, Mortaz E, Karimi K, Kraneveld AD, Rahman I, Caldenhoven E, et al. Cigarette smoke regulates the expression of TLR4 and IL-8 production by human macrophages. J Inflamm (Lond) 2009; 6: 12.

22 Nakahira K, Kim HP, Geng XH, Nakao A, Wang X, Murase N, et al. Carbon monoxide differentially inhibits TLR signaling pathways by regulating ROS-induced trafficking of TLRs to lipid rafts. J Exp Med 2006; 203: 2377-89.

23 Matsuzawa A, Saegusa K, Noguchi T, Sadamitsu C, Nishitoh H, Nagai S, et al. ROS-dependent activation of the TRAF6-ASK1-p38 pathway is selectively required for TLR4-mediated innate immunity. Nat Immunol 2005; 6: 587-92.

24 Zhai C, Yu L, Zhu H, Tian M, Xiaogang Z, Bo W. Porcine CTLA4-lg prolong islet xenografts in rats by downregulating the direct pathway of T-cell activation. Xenotransplantation 2011; 18: 40-5.

25 Labieniec-Watala M, Siewiera K, Jozwiak Z. Resorcylidene aminoguanidine (RAG) improves cardiac mitochondrial bioenergetics impaired by hyperglycaemia in a model of experimental diabetes. Int J Mol Sci 2011; 12: 8013-26.

26 Bocchi L, Savi M, Graiani G, Rossi S, Agnetti A, Stillitano F, et al. Growth factor-induced mobilization of cardiac progenitor cells reduces the risk of arrhythmias, in a rat model of chronic myocardial infarction. PLoS One 2011; 6: e17750.

27 Yu W, Wu J, Cai F, Xiang J, Zha W, Fan D, et al. Curcumin alleviates diabetic cardiomyopathy in experimental diabetic rats. PLoS One 2012; 7: e52013.

28 Kong SS, Liu JJ, Yu XJ, Lu Y, Zang WJ. Protection against ischemiainduced oxidative stress conferred by vagal stimulation in the rat heart: involvement of the AMPK-PKC pathway. Int J Mol Sci 2012; 13: 14311-25.

29 Xu J, Zhou X, Deng Q, Huang Q, Yang J, Huang F. Rapeseed oil fortified with micronutrients reduces atherosclerosis risk factors in rats fed a high-fat diet. Lipids Health Dis 2011; 10: 96.

30 Lv J, Jia R, Yang D, Zhu J, Ding G. Candesartan attenuates Angiotensin II-induced mesangial cell apoptosis via TLR4/MyD88 pathway. Biochem Biophys Res Commun 2009; 380: 81-6.

31 Boudina S, Abel ED. Diabetic cardiomyopathy revisited. Circulation 2007; 115: 3213-23.

32 Codinach Huix P, Freixa Pamias R. Diabetic cardiomyopathy: concept, heart function, and pathogenesis. An Med Interna 2002; 19: 31320.

33 Aydemir-Koksoy A, Bilginoglu A, Sariahmetoglu M, Schulz R, Turan B. Antioxidant treatment protects diabetic rats from cardiac dysfunction by preserving contractile protein targets of oxidative stress. J Nutr Biochem 2010; 21: 827-33.
34 Cosson S, Kevorkian JP. Left ventricular diastolic dysfunction: an early sign of diabetic cardiomyopathy? Diabetes Metab 2003; 29: 455-66.

35 Frustaci A, Kajstura J, Chimenti C, Jakoniuk I, Leri A, Maseri A, et al. Myocardial cell death in human diabetes. Circ Res 2000; 87: 112332.

36 Baraka A, AbdelGawad H. Targeting apoptosis in the heart of streptozotocin-induced diabetic rats. J Cardiovasc Pharmacol Ther 2010; 15 : 175-81.

37 Ho FM, Liu SH, Liau CS, Huang PJ, Lin-shiau SY. High glucose-induced apoptosis in human endothelial cells is mediated by sequential activations of c-Jun $\mathrm{NH}(2)$-terminal kinase and caspase-3. Circulation 2000; 101: 2618-24.

38 Wang GW, Klein JB, Kang YJ. Metallothionein inhibits doxorubicininduced mitochondrial cytochrome $c$ release and caspase-3 activation in cardiomyocytes. J Pharmacol Exp Ther 2001; 298: 461-8.

39 Fiordaliso F, Bianchi R, Staszewsky L, Cuccovillo I, Doni M, Laragione $\mathrm{T}$, et al. Antioxidant treatment attenuates hyperglycemia-induced cardiomyocyte death in rats. J Mol Cell Cardiol 2004; 37: 959-68.

40 Liu ZW, Zhu HT, Chen KL, Dong X, Wei J, Qiu C, et al. Protein kinase RNA-like endoplasmic reticulum kinase (PERK) signaling pathway plays a major role in reactive oxygen species (ROS)-mediated endoplasmic reticulum stress-induced apoptosis in diabetic cardiomyopathy. Cardiovasc Diabetol 2013; 12: 158.

41 Cai L, Li W, Wang G, Guo L, Jiang Y, Kang YJ. Hyperglycemiainduced apoptosis in mouse myocardium: mitochondrial cytochrome C-mediated caspase-3 activation pathway. Diabetes 2002; 51: 1938-48.

42 Johansen JS, Harris AK, Rychly DJ, Ergul A. Oxidative stress and the use of antioxidants in diabetes: linking basic science to clinical practice. Cardiovasc Diabetol 2005; 4: 5.

43 Watanabe K, Thandavarayan RA, Harima M, Sari FR, Gurusamy N, Veeraveedu PT, et al. Role of differential signaling pathways and oxidative stress in diabetic cardiomyopathy. Curr Cardiol Rev 2010; 6: 280-90.

44 Dasu MR, Devaraj S, Zhao L, Hwang DH, Jialal I. High glucose induces toll-like receptor expression in human monocytes: mechanism of activation. Diabetes 2008; 57: 3090-8.

45 Dasu MR, Devaraj S, Park S, Jialal I. Increased toll-like receptor (TLR) activation and TLR ligands in recently diagnosed type 2 diabetic subjects. Diabetes Care 2010; 33: 861-8.

46 Takata S, Sawa Y, Uchiyama T, Ishikawa H. Expression of toll-Like receptor 4 in glomerular endothelial cells under diabetic conditions. Acta Histochem Cytochem 2013; 46: 35-42.

47 Ladefoged M, Buschard K, Hansen AM. Increased expression of tolllike receptor 4 and inflammatory cytokines, interleukin- 6 in particular, in islets from a mouse model of obesity and type 2 diabetes. APMIS 2013; 121: 531-8.

48 Riad A, Jager S, Sobirey M, Escher F, Yaulema-Riss A, Westermann $D$, et al. Toll-like receptor-4 modulates survival by induction of left ventricular remodeling after myocardial infarction in mice. J Immunol 2008; 180: 6954-61.

49 Frantz S, Kobzik L, Kim YD, Fukazawa R, Medzhitov R, Lee RT, et al. Toll4 (TLR4) expression in cardiac myocytes in normal and failing myocardium. J Clin Invest 1999; 104: 271-80.

50 Kawai T, Adachi O, Ogawa T, Takeda K, Akira S. Unresponsiveness of MyD88-deficient mice to endotoxin. Immunity 1999; 11: 115-22.

51 Ha T, Hua F, Li Y, Ma J, Gao X, Kelley J, et al. Blockade of MyD88 attenuates cardiac hypertrophy and decreases cardiac myocyte apoptosis in pressure overload-induced cardiac hypertrophy in vivo. Am J Physiol Heart Circ Physiol 2006; 290: H985-94.

52 Han KJ, Su X, Xu LG, Bin LH, Zhang J, Shu HB. Mechanisms of the 
TRIF-induced interferon-stimulated response element and NF-kappaB activation and apoptosis pathways. J Biol Chem 2004; 279: 1565261.

53 Janssens S, Beyaert R. A universal role for MyD88 in TLR/IL-1Rmediated signaling. Trends Biochem Sci 2002; 27: 474-82.

54 Salaun B, Romero P, Lebecque S. Toll-like receptors' two-edged sword: when immunity meets apoptosis. Eur J Immunol 2007; 37: 3311-8.
55 Hengartner MO. The biochemistry of apoptosis. Nature 2000; 407: 770-6.

56 Huang D, Fang F, Xu F. Hyperoxia-induced up-regulation of Toll-like receptors expression in alveolar epithelial cells. Zhongguo Wei Zhong Bing Ji Jiu Yi Xue 2011; 23: 645-9.

57 Liu ZW, Zhu HT, Chen KL, Qiu C, Tang KF, Niu XL. Selenium attenuates high glucose-induced ROS/TLR-4 involved apoptosis of rat cardiomyocyte. Biol Trace Elem Res 2013; 156: 262-70. 